UCRL-ID-121826

\title{
Rayleigh-Taylor Instability Experiments in a Cylindrically Convergent Geometry
}

\author{
B. Goodwin \\ S. Weir
}

\author{
RECEIVED \\ OCT 061995 \\ OSTI
}

August 25, 1995

This is an informal report intended primarily for internal or limited external distribution. The opinions and conclusions stated are those of the author and may or may not be those of the Laboratory.

Work performed under the auspices of the U.S. Department of Energy by the Lawrence Livermore National Laboratory under Contract W-7405-Eng- 48 .

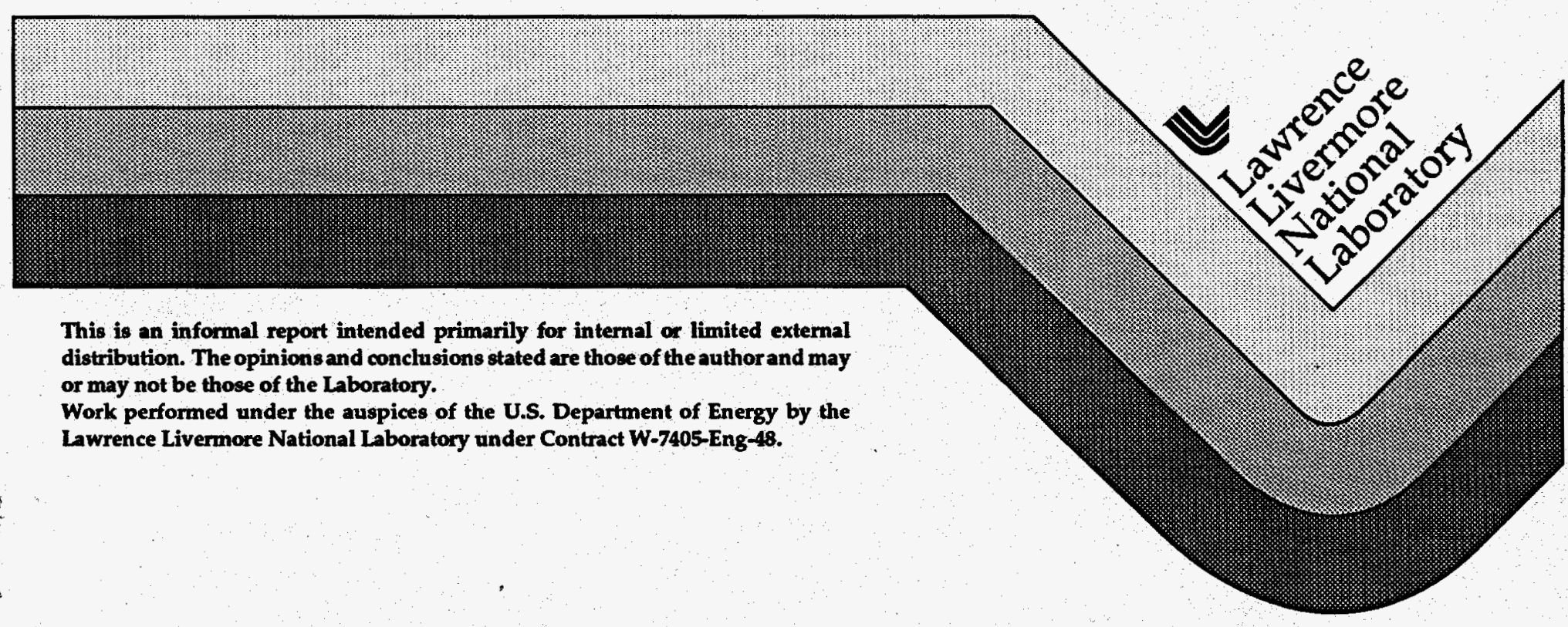

DISTRIBUTION OF THIS DOCUMENT IS UNLIMITED BS 


\section{DISCLAIMER}

This document was prepared as an acccount of work sponsored by an agency of the United States Government. Neither the United States Government nor the University of California nor any of their employees, makes any warranty, express or implied, or assumes any legal liability or responsibility for the accuracy, completeness, or usefulness of any information, apparatus, product, or process disclosed, or represents that its use would not infringe privately own rights. Reference herein to any specific commercial products, process, or service by trade name, trademark, manufacturer, or otherwise, does not necessarily constitute or imply its endorsement, recommendation, or favoring by the United States Government or the University of California. The views and opinions of authors expressed herein do not necessarily state or reflect those of the United States Government or the University of California, and shall not be used for advertising or product endorsement purposes.

This report has been reproduced directly from the best available copy.

Available to DOE and DOE contractors from the Office of Scientific and Technical Information P.O. Box 62, Oak Ridge, TN 37831

Prices available from (615) 576-8401, FTS 626-8401

Available to the public from the

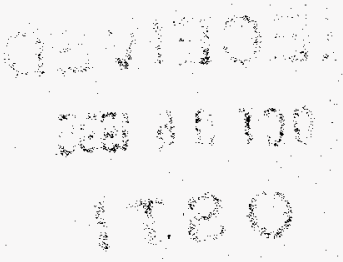

National Technical Information Service

US. Department of Commerce

5285 Port Royal Rd.

Springfield, VA 22161 


\section{DISCLAIMER}

Portions of this document may be illegible in electronic image products. Images are produced from the best available original document. 


\title{
Rayleigh-Taylor Instability Experiments in a Cylindrically Convergent Geometry
}

\author{
B. Goodwin and S. Weir \\ Lawrence Livermore National Laboratory \\ University of California, P.O. Box 808 \\ Livermore, CA 94550
}

\section{Introduction}

Due to the sensitivity of Rayleigh-Taylor instabilities to initial conditions and due to the difficulty of forming well controlled cylindrical or spherical fluid interfaces, Rayleigh-Taylor experiments $12,4,5$ are often performed with simple, planar interfaces. Rayleigh-Taylor instability phenomena of practical interest, however, (e.g., underwater explosions, supernova core collapses ${ }^{6}$, and inertial confinement fusion capsule implosions ${ }^{3}$ ) are typically associated with cylindrical or spherical interfaces in which convergent flow effects have an important influence on the dynamics of instability growth.

Recently, Meshkov et. al. ${ }^{8}$ have developed a novel technique for studying Rayleigh-Taylor instability growth in a cylindrically convergent geometry. Their experiments utilized low-strength gelatin rings which are imploded by a detonating gas mixture of oxygen and acetylene. Since the gelatin itself has sufficient strength to resist significant deformation by gravity, no membranes are needed to define the ring shape. This experimental technique is attractive because it offers a high degree of control over the interfacial geometry and over the material's strength and rigidity, which can be varied by adjusting the gelatin concentration. Finally, since both the gelatin and the explosive product gases are transparent, optical diagnostics can be used.

\section{Experiment}

Our experimental apparatus (Figure 1) is closely modeled on the experiments of Meshkov et. al. . A gelatin ring with an outer diameter of $11 \mathrm{~cm}$, an inner diameter of $8 \mathrm{~cm}$, and a ring thickness of $1 \mathrm{~cm}$ is sandwiched between two 0.5 inch thick plates of Lucite. These Lucite plates are backed by 1.5 inch thick borosilicate glass plates and the whole assembly is secured by means of long steel bolts. The strength and inertial mass supplied by the thick glass plates are important for ensuring that the Lucite-gelatin-Lucite sandwich does not expand appreciably within the time scale of the experiment. Two dimensional hydrodynamic calculations with strength of materials indicate that maximum Lucite wall motion is $\sim 0.01 \mathrm{~cm}$ on the symmetry axis. The inside of the gelatin ring contains air at 1 atmosphere pressure, while the outside of the ring is filled with 1 atmosphere pressure of an equimolar mixture of oxygen and acetylene just prior to the experiment. Gaseous detonation is initiated by bursting of a set of electrical bridge wires (EBW's) with a high current pulse supplied by a high-voltage capacitor. Typically, six or twelve EBW's are symmetrically arranged at a radius of $9.5 \mathrm{~cm}$ from the center of the gelatin ring. 

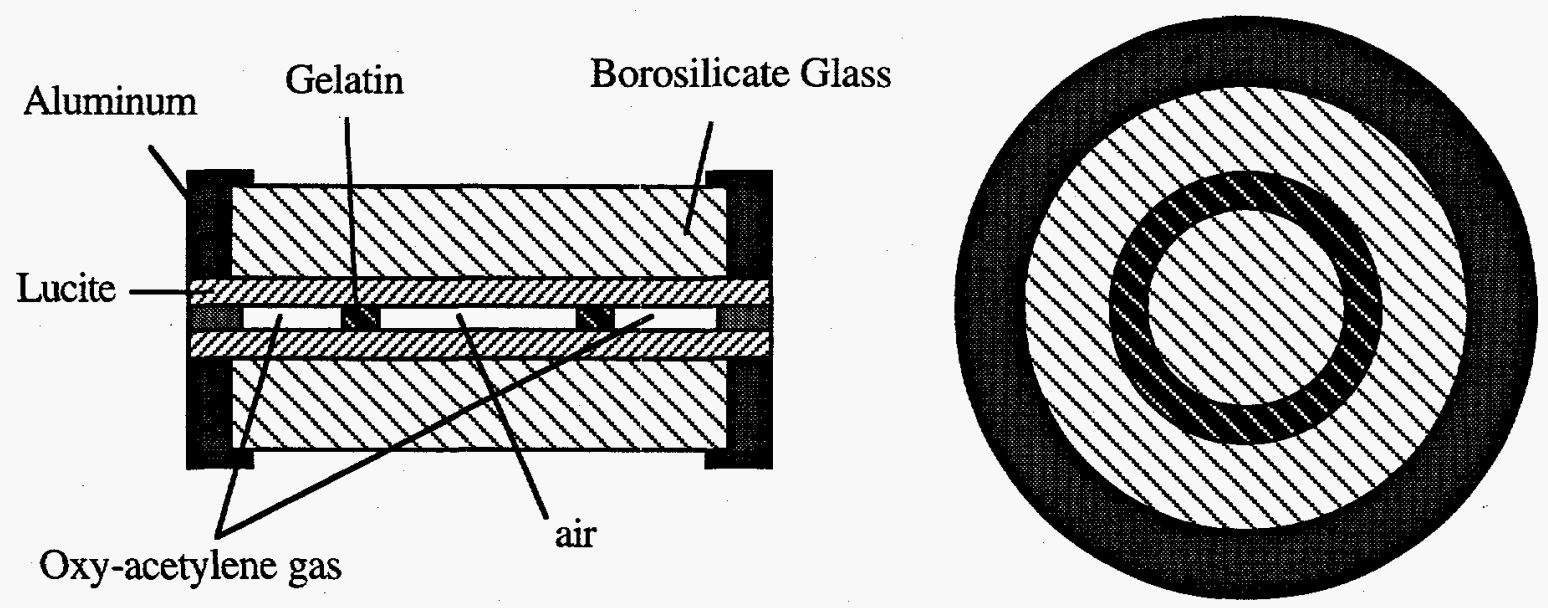

Figure 1. Schematic diagram of experimental fixture for convergent gelatin experiments. Gelatin ring with $11 \mathrm{~cm}$ outer diameter and $8 \mathrm{~cm}$ inner diameter is sandwiched between two Lucite glass plates and imploded by detonation of the oxygen-acetylene gas mixture around the ring.

The gelatin rings were formed using water with a 6-18\% concentration of gelatin (Kind\&Knox Company, 225 Bloom gelatin). A drop of food coloring was also added to improve gelatin visibility. The rings were fabricated by an in situ casting technique, in which the molding fixture was directly fastened onto the actual Lucite plate to be used in the experiment. We found that cast gelatin surfaces were much smoother than gelatin surfaces obtained by cutting. Gelation was at a temperature of $7{ }^{\circ} \mathrm{C}$ for at least 12 hours. Since gelatin strength and rigidity are very sensitive to temperature, the ring was allowed at least 2 hours to equilibrate to room temperature $\left(20^{\circ} \mathrm{C}\right)$ before the experiment. To minimize dragging of the gelatin ring against the Lucite, a small amount of lubricant was applied to the gelatin-Lucite interface .

The equimolar oxygen-acetylene gas mixture was mixed from standard commercial grade gases (99.6\% purity). Due to the extreme spark sensitivity of the mixture ( $<1 \mathrm{~mJ}$ for initiation) special care went into the design and operation of the gas loading system to ensure safety and to avoid premature firing of the system. Gases from separate oxygen and acetylene bottles were fed into a 5 liter mixing chamber, where they were allowed to thoroughly mix before being pumped to the annulus surrounding the gelatin ring. Remotely operated, gas-actuated valves were used to direct the flow of the gases, and to hermetically seal the gelatin fixture just before each experiment. The detonation pressure (Chapman-Jouguet pressure) of an equimolar oxy-acetylene mixture is $45 \mathrm{~atm}$, and the detonation velocity is $0.295 \mathrm{~cm} / \mu \mathrm{s}$.

Optical diagnostics included two high-speed framing cameras (Cordin, Model 6 and Model 121) which, in most experiments, were both oriented to observe the implosions from a position on the ring's axis. The Model 6 operated at an inter-frame speed of $9 \mu \mathrm{sec} /$ frame for a total record length of $1.5 \mathrm{msec}$, and the Model 121 operated at a speed of $8 \mu \mathrm{sec} /$ frame for a total of record length of $0.2 \mathrm{msec}$. The Model 6 camera with its long record length was used to record the entire experiment from initiation until full ring compression, and the Model 121 with its higher resolution optics was used to examine relatively short time intervals of special interest. Illumination was provided by back-lighting the gelatin ring with a high-intensity xenon flashbulb. Control electronics were programmed so that the high-speed cameras were properly synchronized to the xenon flash and the firing of the EBW's. 


\section{Results}

Experiments performed thus far have validated the basic technique and have explored the effects of varying the driving pressure and the gelatin shear modulus. In the experiments presented here, Rayleigh-Taylor instability growth was seeded by (1) the natural surface roughness of the gelatin rings, (2) controlled sinusoidal perturbations fabricated on the gelatin rings, and (3) early-time perturbations impressed onto the outer surface by intersecting detonation and shock waves from the multiple EBW's. Surface roughness measurements have not yet been performed, but from visual inspection of the gelatin rings and the casting molds it appears that the roughness is very small $(<25 \mu \mathrm{m})$.

\section{$\underline{\text { Six initiation-Point Experiments }}$}

\section{A. Axial View Experiment}

Framing camera photographs of an experiment with six EBW initiation points are shown in Figure 2 . The gelatin concentration was $6 \%$. During the early time stages, Rayleigh-Taylor instability growth can be clearly observed on the outer gelatin surface due to both initial surface roughness and early-time perturbations by detonation front interactions. These detonation front interactions result in transient high-pressure pulses at six locations on the ring precisely between each pair of adjacent EBW's. The Rayleigh-Taylor unstable outer surface of the gelatin ring shows a progressively widening dark layer caused by light scattering off the penetrating instability bubbles. Figure 3 shows experimental plots of radial distances vs. time for the outer and inner surfaces of the gelatin ring as well as the position of the instability bubble layer for growth seeded by surface roughness.

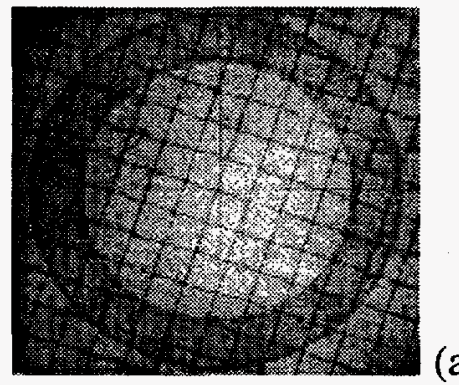

(a)
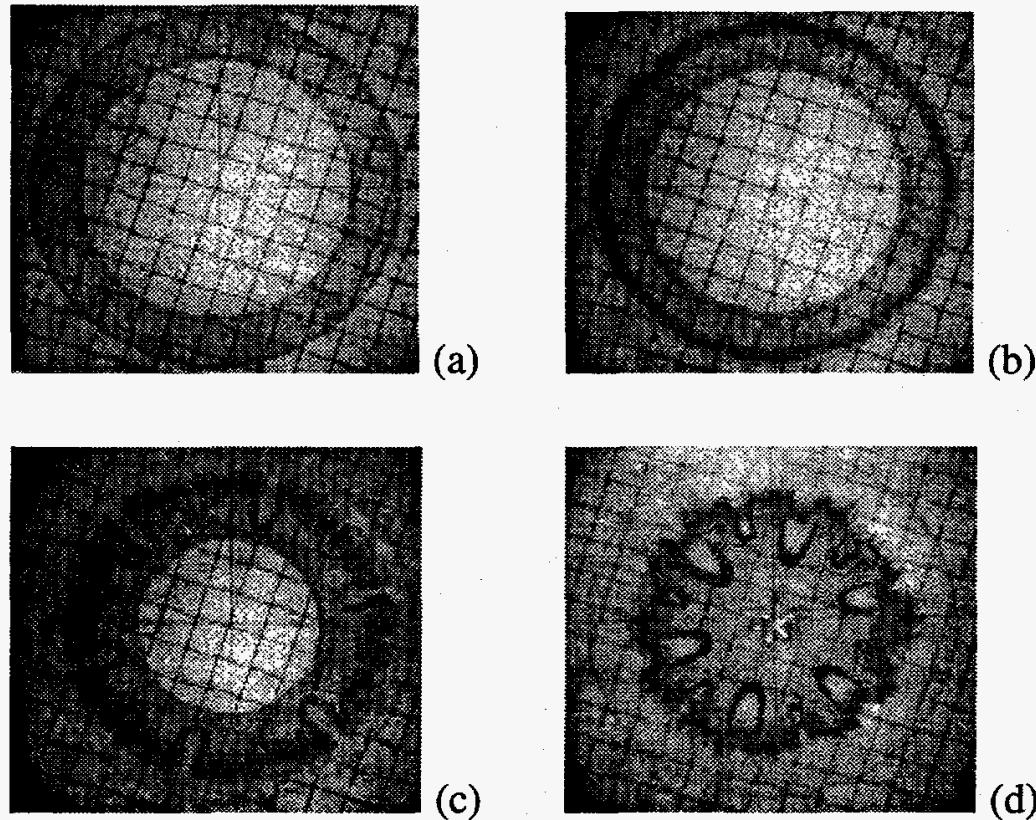

(c)

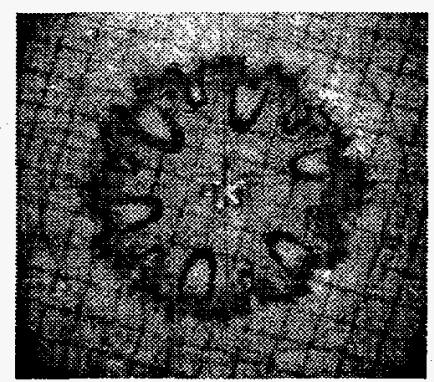

(d)

Figure 2. Gelatin ring experiment with six-point simultaneous initiation of the detonating gas. The grid pattern consists of $1 \mathrm{~cm} \times 1 \mathrm{~cm}$ squares. (a) time $=54 \mu \mathrm{sec}$, (b) $234 \mu \mathrm{sec}$, (c) $504 \mu \mathrm{sec}$, (d) $819 \mu \mathrm{sec}$. 


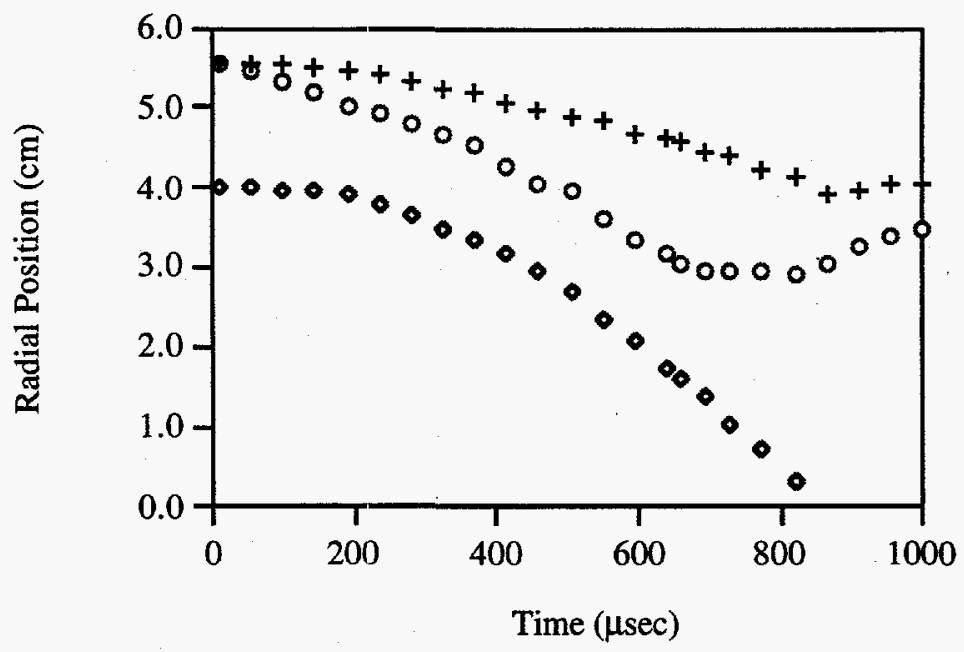

Figure 3. Radial position vs. time for a 6-point gelatin ring experiment. +: Outer boundary of ring. $\bullet$ : Inner boundary of ring. $o$ : Bubble penetration distance.

The initial inward acceleration of the inner boundary of the gelatin ring was observed to be approximately $1.6 \times 10^{-5} \mathrm{~cm} / \mu \mathrm{sec}^{2}$. This initial acceleration decreases during the experiment due to (1) a drop in the explosive gas driving pressure because of adiabatic expansion, and (2) an increase in the air pressure inside the ring because of adiabatic compression. As a result, there is a reversal in the acceleration of the ring from inward to outward at approximately $550 \mu \mathrm{sec}$, at which point the inner boundary becomes Rayleigh-Taylor unstable. However, instability growth on the inner surface is not clearly observable until the large deceleration that occurs around the time of maximum compression of the inner cavity at $820 \mu \mathrm{sec}$. At this point the calculated cavity pressure is about 350 atm.

\section{B. Radial View Experiment}

Experiments were also performed to examine the extent of possible 3-D effects which may cause the motion of the gelatin near the Lucite walls to be significantly different from gelatin at the mid-plane between the two walls. Differences in motion may, for example, be caused by the drag of gelatin on the walls or by enhanced gas driving pressures near the walls because of detonation wave interactions with subsequent gas penetration of the gelatin-Lucite interface. To determine the extent of possible 3-D effects we performed experiments to view the gelatin rings from a radial direction in order to obtain areal images of the outer surfaces of the gelatin rings. For these experiments, glass prisms were mounted inside the experimental fixture of Figure 1 at a position of a few cm outside the gelatin ring so that the sides of the rings could be observed. Figure 4 shows an areal image of the outer surface of a gelatin ring in which six EBW's were used for initiation. The prism was mounted to observe the surface between two adjacent EBW's so that the large instability growth caused by their interacting detonation fronts could be observed. The incipient formation of the bubble due to the interacting fronts can be clearly seen in this image. However, no wall effects are apparent at this time. Thus far, side-view experiments have been performed to approximately $150 \mu \mathrm{sec}$ after detonation initiation, and noticeable 3-D 
effects possibly due to wall dragging can be observed at these times. However, the wall effects still appear to be minor at these times.

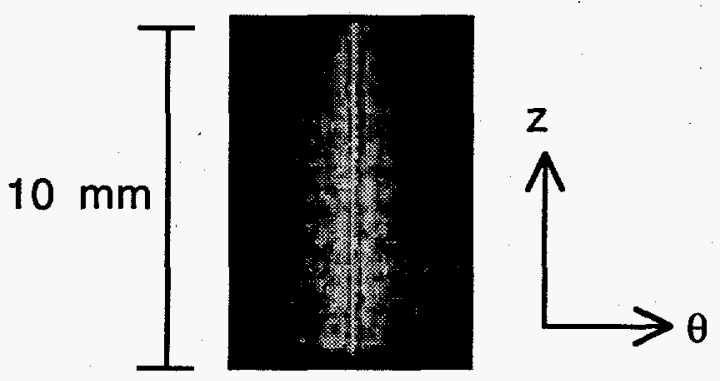

Figure 4. Areal image of gelatin surface in a 6-point experiment. The zdirection is parallel to the axis of the gelatin cylinder and the $\theta$ direction is the azimuthal direction. The time is $24 \mu \mathrm{sec}$ after detonation initiation.

\section{Discussion}

The gelatin ring technique has been demonstrated to produce well controlled instabilities and implosions in a convergent geometry. This technique offers a very high degree of control over the driving pressure and sample strength. In addition, with the casting technique used to fabricate the rings, the geometry of the ring can be precisely controlled in order to seed specific instability modes, so that quantitative experiments of mode mixing in a convergent geometry should be possible.

\section{Six initiation-Point Experiments}

Meshkov et. al. have used the term "turbulent mixing zone" to describe the Rayleigh-Taylor instability zone in the outer surface of the ring in their multi-point initiation gelatin experiments. However, we doubt that this zone, which we have instead called the "instability bubble zone", is a true turbulent mix zone, at least at the gelatin concentrations used thus far ( $>6 \%)$. First of all, the use of a rigid glass apparatus allowed for the recovery of the gelatin rings after the experiments, with the gelatin in all of these experiments being almost entirely intact and undeformed when recovered. This indicates that, for the bulk of the ring, the gelatin yield strength was never exceeded and so turbulence could not have developed. Secondly, the instability zone appears to grow too rapidly to be a turbulent mix zone. According to 2-D numerical simulations of Rayleigh-Taylor instability growth from random short wavelength perturbations, the turbulent mixing zone width should be ${ }^{9}$

$$
h \approx \alpha \frac{\rho_{1}-\rho_{2}}{\rho_{1}+\rho_{2}} g \cdot t^{2}
$$

where $g$ is the acceleration, $t$ is time, and $\rho_{1}$ and $\rho_{2}$ are the densities of the denser and lighter fluids, respectively. The density dependent parameter $\alpha$ is of the order of 0.1 and varies from 0.125 at $\rho_{1} / \rho_{2}=3$ to 0.175 at $\rho_{1} / \rho_{2}=20$. The observed bubble zone width, however, appears to be 
growing at a rate 10 to 20 times faster than predicted by Eqn. 1. We believe that the major perturbations have a saddle surface shape with a pair of large, flat bubbles penetrating the gelatin-Lucite interface connected across the saddle surface along the line of the detonation wave interaction. The width and height of these large flat bubbles can be measured directly. Their depth can be inferred by comparison to perfectly 2-D calculations of the implosion. We modeled the implosion with the 2-D arbitrary lagrangian eulerian code CALE. The CHEETAH thermochemical code provided $\gamma=1.279$ and $\mathrm{E}_{0}=4.46 \mathrm{e}-5 \mathrm{Mb}-\mathrm{cm}^{3}$ for the equimolar gas mixture, with the detonation velocity measured at $0.295 \mathrm{~cm} / \mu \mathrm{s}$.

Figure 5 shows both the measured and calculated inner, outer and average radii versus time during the implosion. By assuming a 2-D implosion, these radii can be turned into calculated and measured volumes versus time (figure 6). As one would expect for a highly incompressible material such as water gelatin, the calculated 2-D implosion shows essentially no change in volume during the implosion. The measured radii show an apparent $20 \%$ volume increase which peaks at the time of implosion bounce and then decreases to the original value. We assume that this apparent increase is the result of gas injection at the gelatin-Lucite interface. The injected gas bubbles cause the inner and outer surfaces of the gelatin to be moved inward and outward with respect to the calculated radii (as seen in fig. 5) in order to compensate for the included volume of gas products. The average thickness of these interface bubbles can be estimated by noting that the large bubbles are $\sim 1 . \times 2 . \mathrm{cm}$ and the smaller bubbles are $0.5 \times 1 . \mathrm{cm}$ in diameter. There are twelve of each in the 6 point initiated experiment. Neglecting the included volume of unresolved fine bubbles (i.e. all of the excess $7.5 \mathrm{~cm}^{3}$ of volume is due to the major interface bubbles) we estimate the thickness to be less than $\sim 0.3 \mathrm{~cm}$.

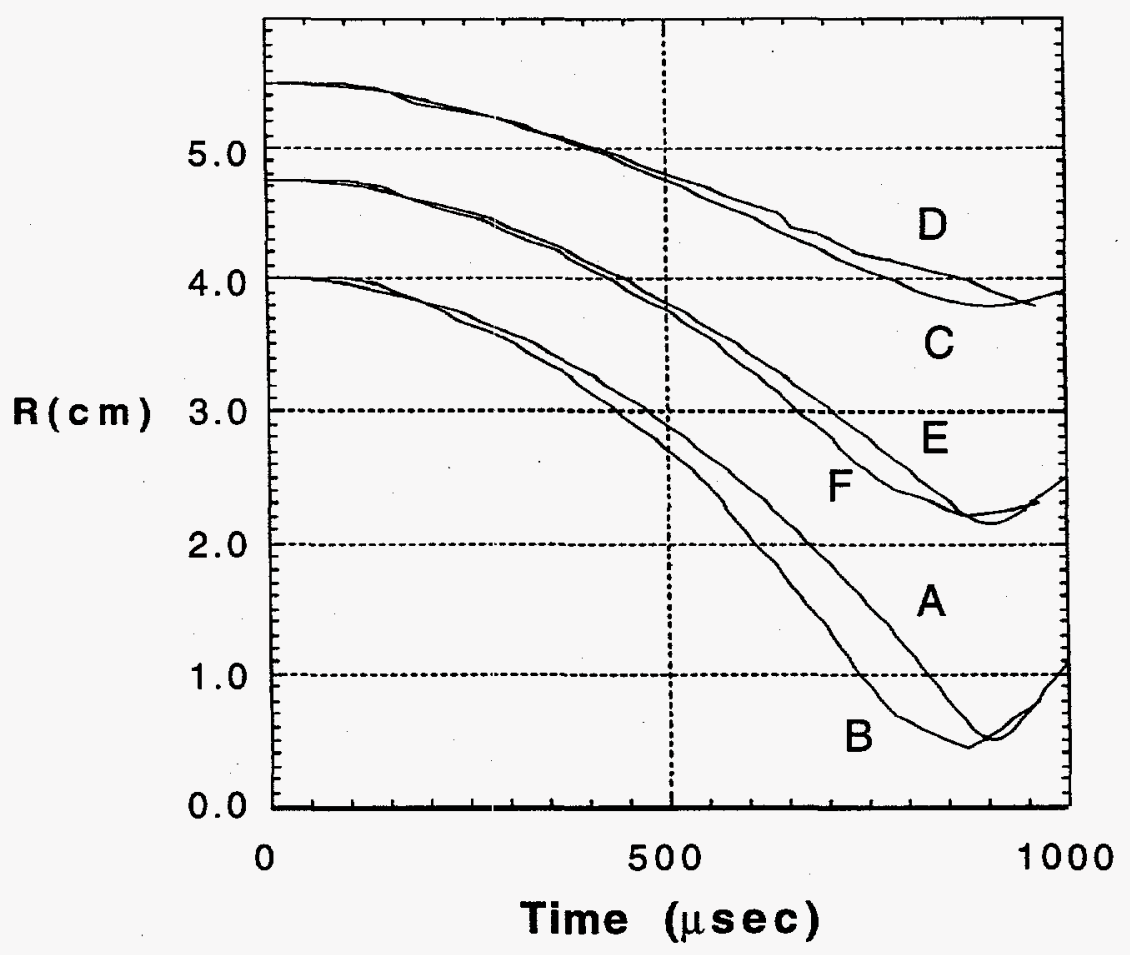

Figure 5. A \& C: calculated inner and outer radii (cm) vs. time ( $\mu \mathrm{s})$. B \& D : measured inner and outer radii vs. time. E \& F: calculated and measured average radii vs. time ( $6 \%$ gelatin, 0.25 bars shear modulus) 


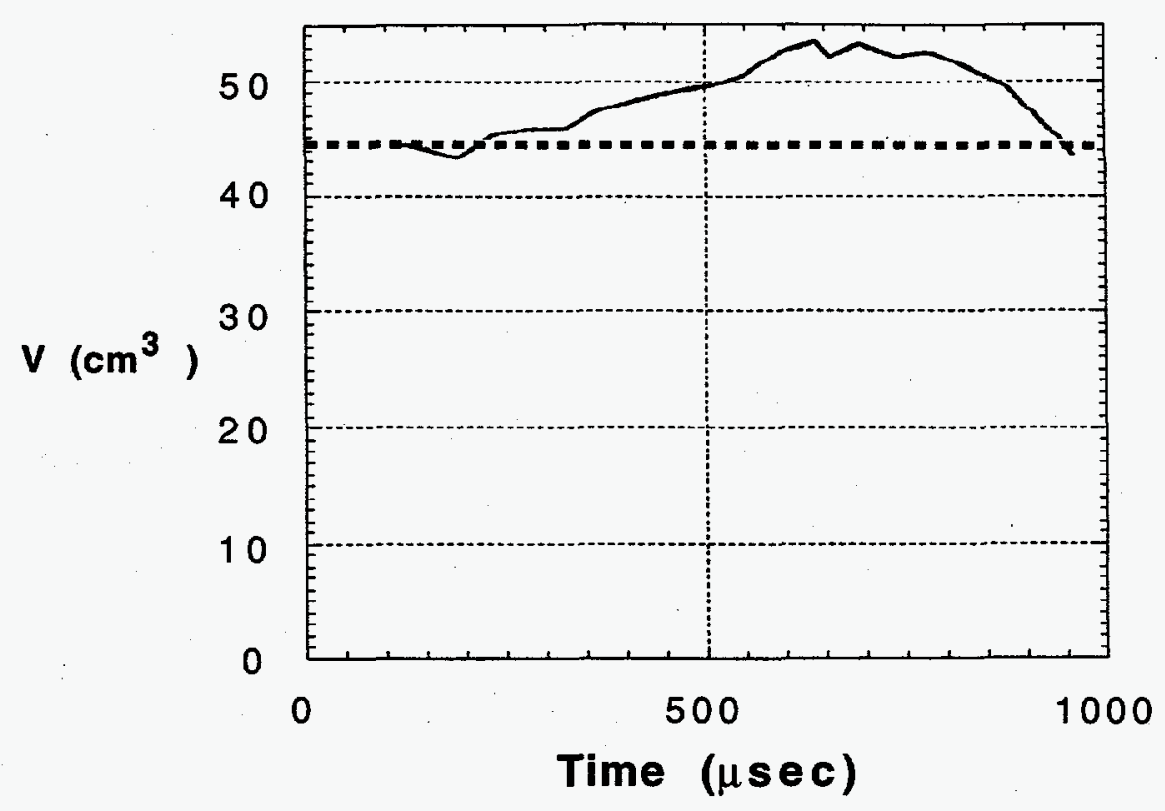

Figure 6. Volume vs. time for gelatin ring. Dotted Line: calculated volume of gelatin. Solid Line: apparent measured volume of gelatin inferred from Fig. 5.

2-D calculations with initial perturbations at the gelatin-Lucite-gas intersection point do show gas penetrating the gelatin-Lucite interface. The experimentally observed bubbles are highly 3-D and so we plan to pursue fully 3-D calculations of these phenomena.

\section{Sinusoidal Perturbation Experiments}

To study the growth of instabilities when small initial perturbations are present, we performed experiments in which small sinusoidal waves were fabricated on the outer circular surfaces of the gelatin rings. Figure 7 shows the results of an implosion initiated at one point with a ring having a 36 mode, $0.1 \mathrm{~cm}$ amplitude initial perturbation molded onto the outer gelatin surface. A preliminary calculation of this experiment illustrated in Fig. 8 shows reasonable fidelity to the experimental result. Pre-emplaced, molded perturbations are more controllable than those determined by the detonation pattern. The simple detonation structure of a single initiation point seems to reduce interface gas penetration. The measured gas detonation velocity $(0.294$ $\mathrm{cm} / \mu \mathrm{s})$ is an order of magnitude larger than the calculated peak outer radial velocity $(\sim 0.026$ $\mathrm{cm} / \mu \mathrm{s})$. We can therefore initiate the gas detonation at a single point and obtain an essentially symmetric implosion. We are currently exploring techniques to obtain simultaneous circumferential gas initiation in order to eliminate the transverse flows observable in Figures 7 and 8 . We also will modify the geometry of the ring so as to greatly reduce or eliminate interface penetration effects. 


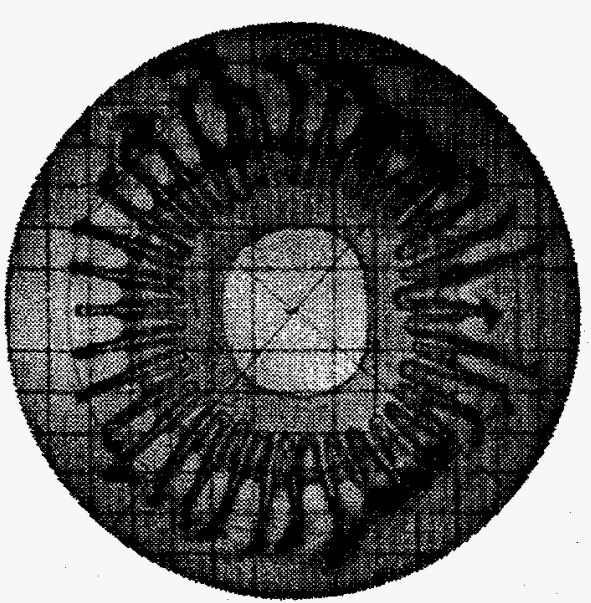

(a)

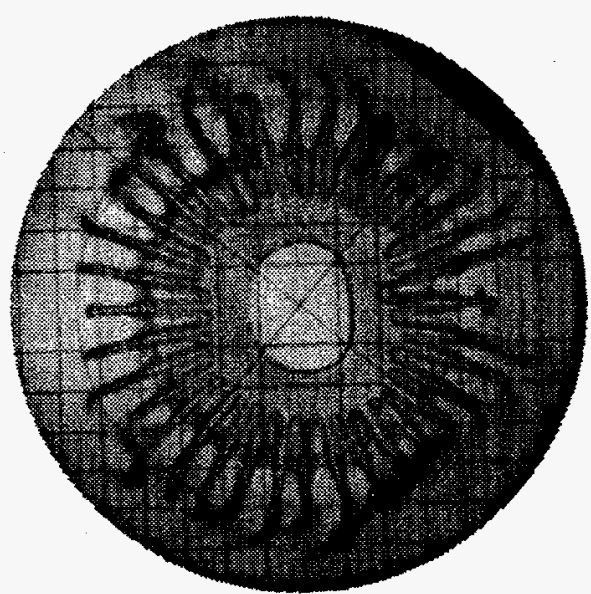

(b)

Figure 7. Molded 36 mode, $0.1 \mathrm{~cm}$ amplitude sinusoidal perturbation experiment with single point detonation initiation. (a) time $=630 \mu \mathrm{sec}$.

(b) time $=675 \mu \mathrm{sec}$.

(a) $620 \mu \mathrm{sec}$

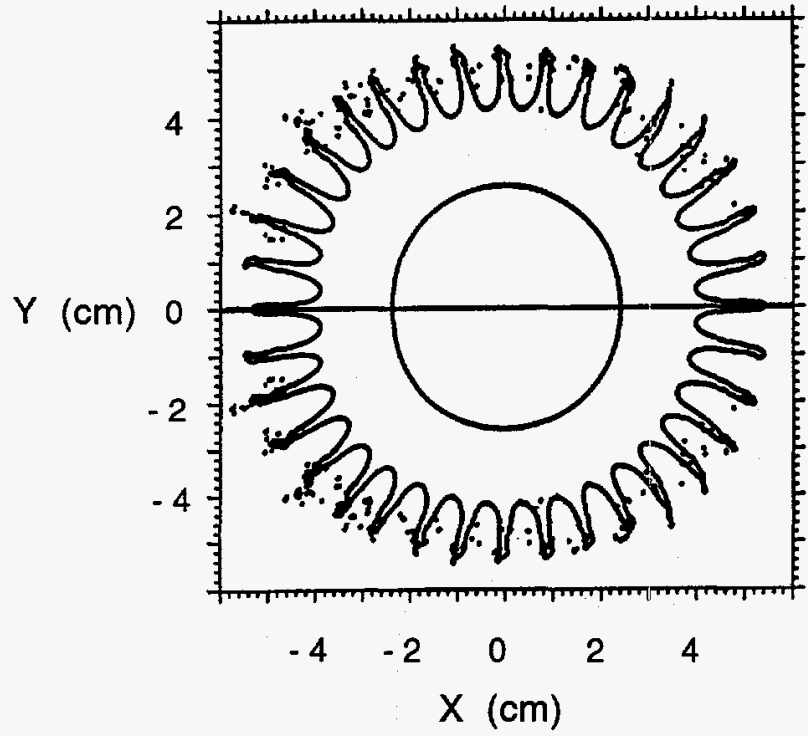

(b) $700 \mu \mathrm{sec}$

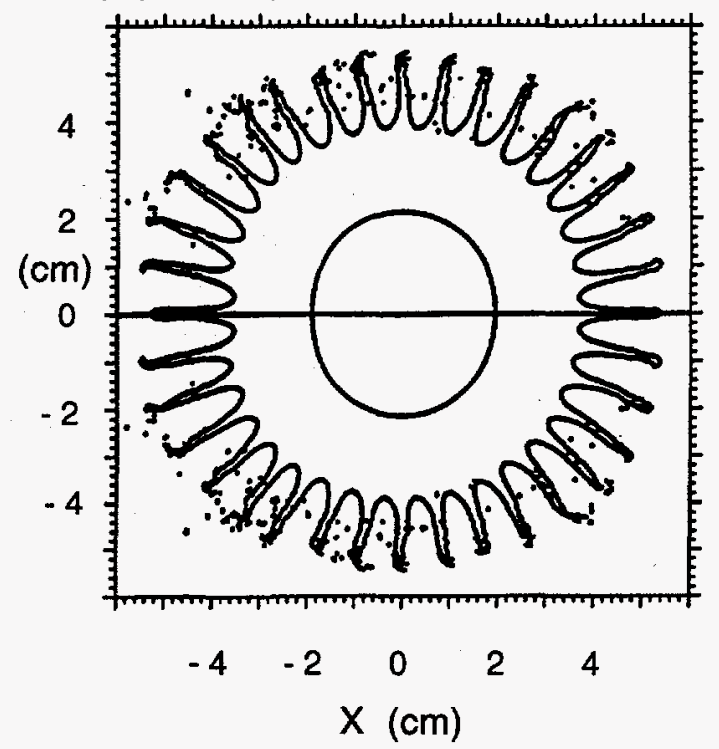

Figure 8. CALE 2-D calculation of molded 36 mode, $0.1 \mathrm{~cm}$ amplitude sinusoidal experiment with single point detonation initiation at $x=9.5 \mathrm{~cm}$, $y=0$. cm. (a) time $=620 \mu \mathrm{sec}$. (b) time $=700 \mu \mathrm{sec}$.

\section{Acknowledgments}

We would like to acknowledge the efforts and contributions from the experimental team of D. Anderson, B. Gray, D. Burns, A. Hernandez, and K. Moua. We also thank J. Chase for valuable discussions and would like to acknowledge the efforts of J. Chase and C. Clements in establishing the safety procedures for these experiments. 


\section{References}

M. Ratafia, Phys. of Fluids 16 ,1207 (1973).

R. Popil and F.L. Curzon, Rev. Sci. Instrum. 50, 1291 (1979).

3 R.L. Cole and R.S. Tankin 16, 1810 (1973).

4 D.J. Lewis, Proc. Royal Soc. Lond. A202, 81 (1950).

5 K.I. Read, Physica 12D, 45-58 (1984).

6 L. Smarr, J.R. Wilson, R.T. Barton, and R.L. Bowers, Astrophys. J. 246, 515 (1981).

7 E.M. Campbell, Laser and Particle Beams 9, 209 (1991).

8 E.E. Meshkov, N.V. Nevmershitsky, V.G. Rogachev, and I.G. Zhidov, Proc. 4th Int. Workshop on the Phys. of Comp. Turb. Mixing, Cambridge, England 578 (1993).

9 D.L. Youngs, unpublished. 